\title{
The Structure and Interaction History of Moderate Luminosity Mergers
}

\author{
Susanne Hüttemeister ${ }^{1}$ and Eva Manthey ${ }^{2}$ \\ ${ }^{1}$ Astronomisches Institut Ruhr-Universität Bochum, D-44780 Bochum, Germany \\ email: huette@astro.rub.de \\ ${ }^{2}$ ASTRON, NL-7880AA Dwingeloo, The Netherlands \\ email: manthey@astron.nl
}

\begin{abstract}
Interaction and merging are the two most important driving forces of galaxy evolution. In contrast to the well studied major mergers of two large gas-rich disk galaxies, which may lead to Ultraluminous Infrared Galaxies (ULIRGs, $\mathrm{L}_{\mathrm{FIR}}>10^{12} \mathrm{~L}_{\odot}$ ), mergers between galaxies of unequal mass or a spiral and an elliptical ( $\mathrm{S}+\mathrm{E}$ mergers) are still poorly understood. These moderate luminosity mergers typically have $\mathrm{L}_{\mathrm{FIR}}<10^{11} \mathrm{~L}_{\odot}$. Here, we present the results of our multiwavelength study of a sample of such kind of mergers, including interferometric $\mathrm{HI}$ and single-dish CO observations, optical and near-infrared imaging as well as optical spectroscopy.
\end{abstract}

Keywords. galaxies: interactions, evolution, starburst

\section{The Project \& Results}

One of the most essential questions connected to these $\mathrm{S}+\mathrm{E}$ mergers is the nature of the starburst the merger induces. There is very significant disagreement between results of numerical simulations. The key question is the degree of gas concentration that is reached in the merging process. Weil \& Hernquist suggest a strong central concentration of the gas in a minor merger (similar to ULIRGs), while calculations by Kojima \& Noguchi (1997) indicate a 'scattering' of gas on large scales, possibly not resulting in a starburst at all, since the gas will become too dispersed in the collision. Based on the results of the prototypical S+E merger, NGC 4194 (the Medusa), which hosts an extended starburst and correspondingly an extended molecular gas reservoir (Aalto \& Hüttemeister (2000)), none of the two models can be favoured. To study the properties of $\mathrm{S}+\mathrm{E}$ mergers more generally, we obtained a systematic multiwavelength investigation of a sample of $15 \mathrm{~S}+\mathrm{E}$ merger candidates. A detailed analysis of all data is given in Manthey (2006). A number of characteristics of this class of mergers emerge: Moderate luminosity mergers seem to be characterised by an extended region of enhanced star formation (SFR up to $40 \mathrm{M}_{\odot} / \mathrm{yr}$ ), typically several kpc in size, i.e. considerably larger than what is found in a ULIRG. Even more striking, the large scale HI distribution, which is often very complex and consists of numerous tidally induced structures, like tails, plumes and shells, bears almost no resemblance to the optical appearance of the merger remnant. This points to an origin of moderate luminosity mergers that is distinctly different from ULIRGs, where the atomic gas usually at least roughly follows the optical features.

\section{References}

Aalto, S. \& Hüttemeister, S., 2000, $A \& \mathcal{E} A 362,42$.

Kojima, M. \& Noguchi, M., 1997, ApJ 481, 132.

Manthey, E., 2006, PhD thesis, AIRUB.

Weil, M.L. \& Hernquist, L., 1993, ApJ 405, 142. 